\title{
Plant Pillow Preparation for the Veggie Plant Growth System on the International Space Station
}

\author{
Gioia D. Massa ${ }^{1}$, Gerard Newsham², Mary E. Hummerick ${ }^{3}$, Robert C. Morrow ${ }^{4}$, and Raymond M.
} Wheeler $^{1}$

${ }^{I}$ NASA, Kennedy Space Center, FL; ${ }^{2}$ TOSC Team, Jacobs, Kennedy Space Center, FL; ${ }^{3}$ Chemical and Biological Sciences Labs, Engineering Services Contract, Kennedy Space Center, FL; ${ }^{4}$ ORBITEC, Madison, WI

\begin{abstract}
The first Veggie plant growth chamber was installed on the International Space Station in 2014. Crop plants can be grown in Veggie using plant pillows, small rooting packets that contain substrate, fertilizer, and germination wicks along with attached seeds. The pillows were designed to interface with the Veggie root mat reservoir watering system to provide a capillary water column to growing plants. In preparation for flight, methods of arcillite substrate washing, autoclaving, and drying were established to reduce dust and to provide a dry sterile substrate. A controlled released fertilizer mixed into arcillite substrate provides nutrition for plant growth. Methods of seed surface sterilization were tested for both germination and microbial contamination, and the optimum methods were determined for candidate flight crops. Plant pillows were prepared for flight by cutting and inserting germination wicks, filling with the
\end{abstract}

Key words: Arcillite; Controlled Release Fertilizer; Food Production; International Space Station; Plant Pillows; Veggie; Vegetable

Correspondence to: Gioia D. Massa Mail Code UB-A

Kennedy Space Center, FL 32899

Telephone: 321-861-2938

E-mail: gioia.massa@nasa.gov substrate/fertilizer mix, and sewing closed. Following pillow filling, seeds were attached to the wicks, and the pillows were packaged for flight. Pillow preparation methods have been successfully tested in the VEG-01 hardware validation tests on the International Space Station with 'Outredgeous' lettuce and 'Profusion' zinnia, and in the VEG-03 test, using 'Outredgeous' lettuce and 'Tokyo bekana' Chinese cabbage.

\section{INTRODUCTION}

Veggie is an expandable plant chamber designed and built by ORBITEC (Madison, WI) for the International Space Station (ISS). The chamber provides approximately $0.13 \mathrm{~m}^{2}$ growing area with a red, green, and blue LED lighting system. A recent review (Massa et al., 2016) provides a more detailed description of this plant growth hardware, focusing primarily on the LED lighting array. NASA launched the Veggie chamber to the ISS in April, 2014, and the chamber was installed and tested beginning in May, 2014 as part of the VEG-01 hardware validation test. Veggie was designed with a root mat reservoir that originally was intended to provide both water and nutrients to support the growth of vegetable crops (Morrow et al., 2005; Morrow and Remiker, 2009). Testing by Stutte et al. (2011) documented the buildup of salt and algal/microbial growth on the rooting mats when nutrient solution was used in the reservoir. These 
issues could be mitigated by mixing controlled released fertilizer into a solid substrate contained in rooting packets, and using the reservoir to provide water to these packets via capillary action. This work led to the development of the current Veggie plant pillow, an expandable, gusseted Teflon ${ }^{\mathrm{TM}}$ coated Kevlar ${ }^{\circledR}$ bag, manufactured by ORBITEC. The pillows have a Nomex ${ }^{\circledR}$ wicking bottom surface that interfaces directly to the Veggie root mat reservoir, designed to establish a capillary column from the reservoir to the rooting substrate. Each pillow contains a silicon foam gasket penetrating the top surface with a central slot to allow the insertion of wicks and seeds. This gasket allows substrate containment while plant shoots can emerge and enlarge without constraint. Plant pillows were designed in different sizes to allow for different plant types to be grown. Number 6 (\#6) pillows (six per a 0.13 $\mathrm{m}^{2}$ Veggie) hold $250 \mathrm{~cm}^{3}$ of substrate and can be used for smaller crops like leafy vegetables. Number 3 (\#3) pillows hold $500 \mathrm{~cm}^{3}$ of substrate, while \#2 pillows hold $750 \mathrm{~cm}^{3}$ of substrate. These larger volume pillows could be used for medium crops such as dwarf tomatoes or dwarf peppers. Number 1 (\#1) pillows were designed so that one pillow encompassed an entire Veggie area. These would hold $1500 \mathrm{~cm}^{3}$ of substrate and could be used for longer duration crops requiring large root zones, such as dwarf fruit trees (e.g., Graham et al., 2015).

As initial validation flight tests of the Veggie hardware were being planned, methods for use of the Veggie pillows needed to be established. Three primary areas of methods development were required - substrate preparation, seed preparation, and pillow preparation. One flight safety criterion was that the pillow substrates would be free of dust and contaminant free before flight. Therefore, methods of cleaning to remove dust and to generate a sterile substrate for plant growth were established. A second requirement imposed upon the Veggie system was that seeds would be surface sterilized prior to planting in the pillows to reduce the potential incidence of plant disease or contamination on the ISS. Surface sterilized seeds also had to maintain viability for extended durations of dry storage after treatment, because the VEG-01 and VEG-03 series of tests had no definite start or end dates when launched. Plant varieties tested had differential responses to surface sterilization methods, and responses to these methods helped drive crop selection for VEG-01. Finally, methods were developed to pack and plant the pillows in a clean environment. These planting and packaging techniques had to be tested to show that substrates and seeds remained in place and viable after launch. The methods below discuss the preparation of the plant pillows for both the VEG-01 initial validation test and the VEG-03 second crop test series in the Veggie hardware. Observed on-orbit plant growth results of these tests are discussed further by Massa et al. (2017).

\section{MATERIALS AND METHODS}

\section{Substrate Preparation}

Veggie plant pillows were filled with two different sized arcillite substrates that were down selected from previous ground testing (Massa et al., 2013) as well as other spaceflight investigations (e.g., Morrow et al., 1994; Morrow and Crabb, 2000; Morrow et al., 2001; Jones et al., 2002; Norikane et al., 2005). Half of the plant pillows flown for both VEG-01 and VEG-03 used a substrate with particles ranging from $600 \mu \mathrm{m}$ to $1 \mathrm{~mm}$. The remainder used a blend of $50 \% 600$ $\mu \mathrm{m}$ to $1 \mathrm{~mm}$ and $50 \% 1-2 \mathrm{~mm}$ particles. Data from the VEG-01 and VEG-03 investigations will be used to ascertain if either substrate will perform better for the growth of leafy greens in microgravity, but to date significant differences have not been observed. For VEG-01 and VEG03 , each substrate was used in conjunction with Nutricote, a polymer coated controlled release fertilizer (18-6-8, type 180, Florikan, Sarasota, FL) at rates of $7.5 \mathrm{~g} / 1000 \mathrm{~cm}^{3}$ dry substrate as previously tested (Stutte et al., 2011; Massa et al., 2013). Prior to mixing, methods for removal of dust and any contaminants from the substrate needed to be established.

\section{Substrate washing}

Arcillite substrate (Turface Proleague, Profile Products, LLC) was obtained at two particle sizes $-600 \mu \mathrm{m}$ to $1 \mathrm{~mm}$ and $1-2 \mathrm{~mm}$. The substrate was sieved using standard mesh sieves collecting the fraction between mesh 18 and mesh 30 (600 $\mu \mathrm{m}$ to $1 \mathrm{~mm}$ ) and the fraction between mesh 10 and mesh $18(1-2 \mathrm{~mm})$. The arcillite substrate contained significant levels of dust, which had to 
be removed prior to flight for both crew safety and to maintain functionality of the hardware by reducing the potential for particulate clogging on the reservoir surface and in the fan. To remove dust, two methods were tested for substrate washing. The original method was used in VEG01, and an upgraded method was added for VEG03. For the original substrate washing method, the substrate was washed multiple times in a $30 \mathrm{~L}$ substrate washer (modified from a design suggested by ORBITEC, see Figure 1) using deionized $\mathrm{H}_{2} \mathrm{O}$ to remove adhering dust. The substrate washer consists of a $30 \mathrm{~L}$ Nalgene $^{\mathrm{TM}}$ Heavy-Duty Cylindrical LLDPE Tank with a spigot (Fisher Scientific, Pittsburgh, PA), three pairs of well washed standard bricks (Lowe's, Mooresville, North Carolina), and two $25.4 \mathrm{~cm}$ standard mesh sieves (Fisher Scientific, Pittsburgh, PA). Using this system, substrate was washed in $1000 \mathrm{~cm}^{3}$ batches, with $500 \mathrm{~cm}^{3}$ in each of two sieves in the substrate washer. For the 600 $\mu \mathrm{m}$ to $1 \mathrm{~mm}$ substrate, arcillite was placed in a $600 \mu \mathrm{m}$ (\#30) sieve. For the 1-2 mm substrate, substrate was placed in a $1 \mathrm{~mm}$ (\#18) sieve. The $30 \mathrm{~L}$ tank was filled with deionized water $(24 \mathrm{~L}$ with displacement from bricks, sieves, and substrate) with water flowing over the surface of the substrate to remove dust, and then the water in the tank was drained. This process was cycled an average ten times until drain water exiting the spigot was visibly clear plus one additional rinse. Differences in substrate dustiness led to different numbers of required rinses per batch of substrate. Visible clarity was assessed by sampling outflow water and viewing it with backlighting. Visible clarity was judged as the time when no turbidity or particles were observed.

The second, upgraded washing method consisted of using the same sieves positioned on bricks over a sink drain with a custom washing unit positioned over the sieves (Figure 2). Deionized $\mathrm{H}_{2} \mathrm{O}$ was run through the sieves via two shower head attachments (Aqua Dance 8 Rainfall Showerhead, Target, Minneapolis, MN) composed of plastic and stainless steel to eliminate any potential corrosion products leaching in to the substrate. Shower heads were supported with an 80/20 aluminum and PVC frame allowing heads to be raised or lowered. Each shower head is capable of operating independently, or both can be operated simultaneously using PVC shut off valves. Water was run through $250 \mathrm{~cm}^{3}$ of arcillite substrate in each sieve until visibly clear drainage water was observed using the established protocol (see above), with confirmation made from a catch beaker held under the sieve outflow. The substrate was stirred once a minute during a wash.

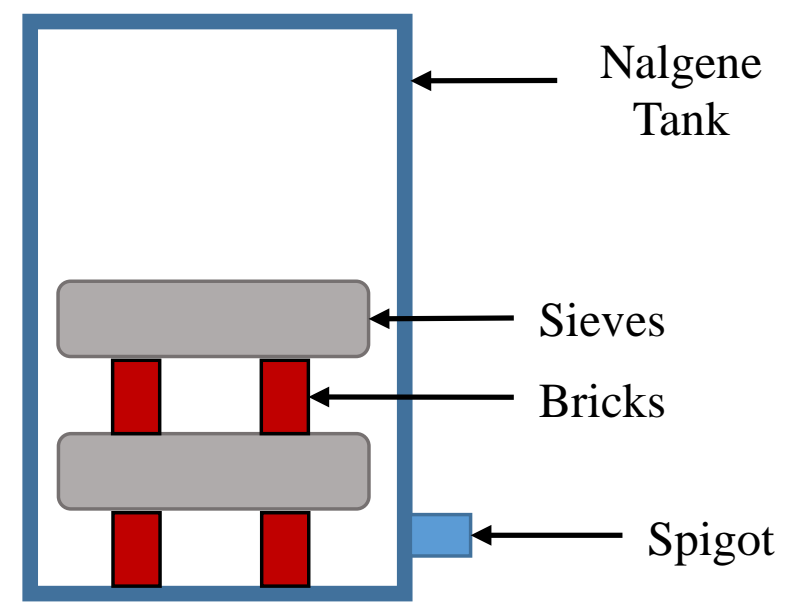

Figure 1. Schematic for substrate washing tank modified from original design of Robert Morrow, ORBITEC.

\section{Substrate finishing}

Following washing with either method, wet and washed substrate was placed in polypropylene sterilizing pans (14.2 L, Thermo Fisher Scientific, Rochester, NY), covered with aluminum foil, and autoclaved for a $60 \mathrm{~min}$ gravity cycle with a 30 min dry cycle to eliminate any biological contamination. Pans were removed from the autoclave and oven dried to dryness in a forced air oven at $70^{\circ} \mathrm{C}$ for at least $72 \mathrm{~h}$ without removing covering foil. Substrate was allowed to cool to room temperature and then, within a calibrated sterile laminar flow hood or microbiological safety cabinet, it was transferred to a sterile reclosable bag. Prepared substrate was stored until needed. Just prior to pillow filling operations, substrate was carefully mixed in a sterile reclosable bag in 1000-2000 $\mathrm{cm}^{3}$ batches with added fertilizer. Substrate was mixed by placing measured volumes of substrates and fertilizer in an unused reclosable bag, sealing, and gently agitating to ensure mixing but to minimize any arcillite degradation and dust formation. 


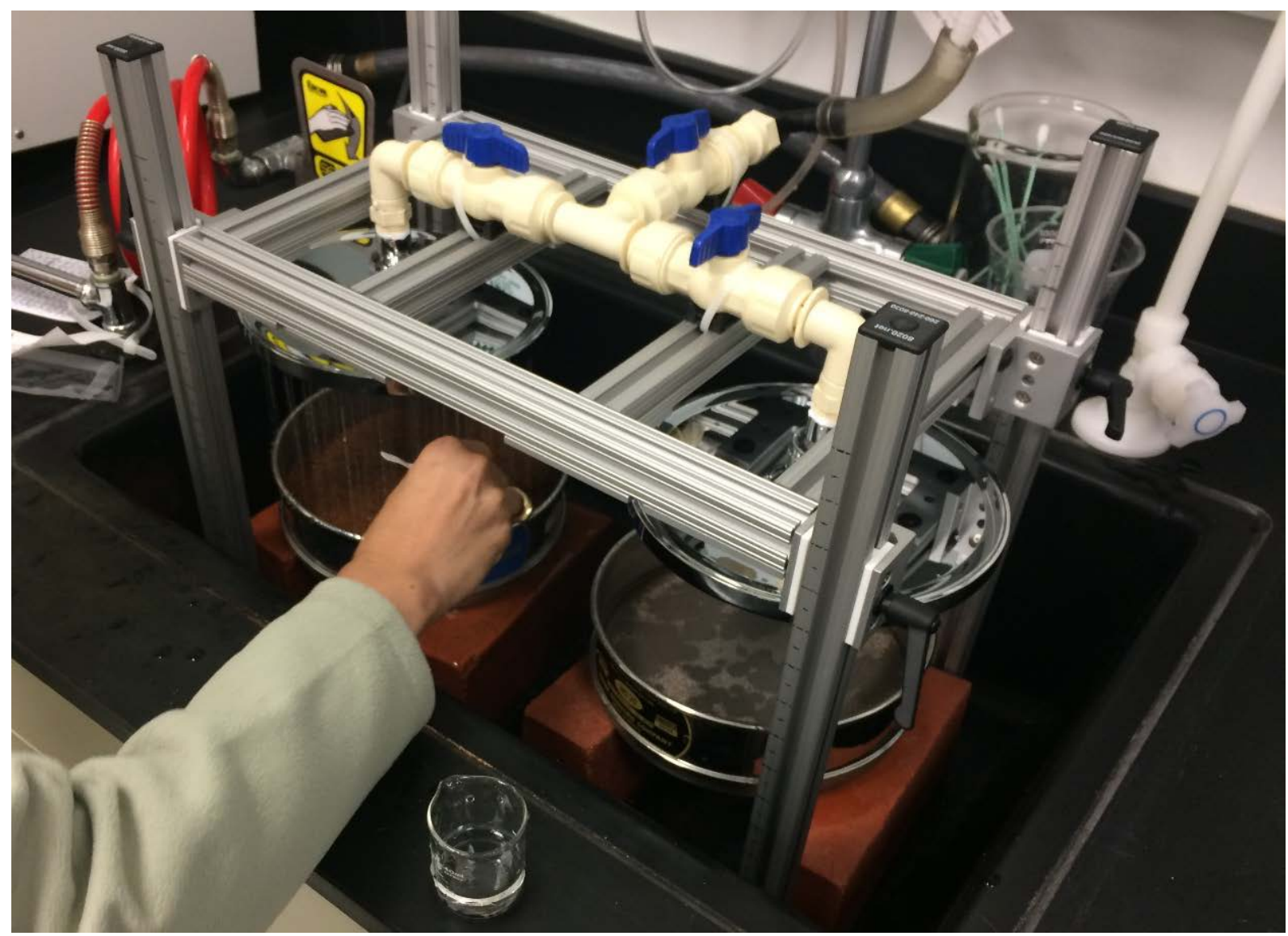

Figure 2. Custom substrate washer built at the Kennedy Space Center, FL.

Mixing volumes of $1000-2000 \mathrm{~cm}^{3}$ substrate with fertilizer in this method with careful observation of fertilizer distribution provided uniformity of fertilizer in the plant pillows. Plant growth tests prior to flight confirmed that plants in pillows filled with this method had sufficient nutrient levels. For VEG-01 and VEG-03, the fertilizer utilized was Nutricote controlled release fertilizer (18-6-8, type 180, Florikan, Sarasota, FL) at a rate of $7.5 \mathrm{~g} / 1000 \mathrm{~cm}^{3}$ dry substrate. Fertilizer was not sterilized prior to flight as sterilization procedures could damage the polymer coating. To keep the fertilizer source clean and free of contaminants, the bag of fertilizer was opened only in a controlled laboratory environment and maintained in a controlled clean container.

\section{Seed Preparation}

VEG-01 focused on one leafy green crop, 'Outredgeous' red romaine lettuce (Lactuca sativa cv. 'Outredgeous'), which would be grown twice to allow food safety data to be collected from the first crop and produce consumption to be tested with the second crop, assuming the first was shown to be safe to consume. A third, ornamental crop was proposed to test flowering and initial plans called for a combination of 'Profusion' zinnia (Zinnia hybrida cv. 'Profusion' mix) and 'Durango' French marigold (Tagetes patula cv. 'Durango' Outback mix). VEG-03 focused on leafy green crops, with one set of 'Outredgeous' lettuce and two sets of 'Tokyo Bekana' dwarf Chinese cabbage (Brassica rapa var. chinensis) selected for flight to look at different harvest scenarios of these crops. Seed responses to surface sterilization methods were assessed and two separate seed surface sterilization methods were developed to accommodate different seed types. Testing focused on methods with no or minimal water to reduce chances of seed imbibition leading to poor germination after long 
duration storage. A series of tests were run. Initially, bleach fuming alone, followed by a bleach-based chlorine fuming technique, modified from Clough and Bent (2011), were tested. Different concentrations of chlorine and treatment durations were tested. A method was determined that was shown to successfully surface sterilize lettuce without inhibition of immediate and stored seed germination. Additionally, an ethanol submersion technique was developed and tested, modified from Maloof (2014).

\section{Chlorine fuming}

The appropriate numbers of seeds were counted and $\sim 15 \%$ additional seeds were added, and placed in a $60 \mathrm{~mm}$ Pyrex Petri dish. A $50 \mathrm{~mL}$ Pyrex beaker was inverted in a Ball Jar (regular mouth 8 oz. glass Mason jar, Ball and Kerr, Fishers, IN). Thirty $\mathrm{mL}$ of household bleach (5.25\% sodium hypochlorite) was added and it was sealed with the two-part jar lid. The jar and the dish of seeds were transferred to a fume hood. Initial (bleach alone) fuming was tested by placing a dish of seeds in the jar on the beaker and sealing it for a test duration (Table 1). Later tests used concentrated $\mathrm{HCl}$ added to the bleach to fume the seeds with chlorine gas. For chlorine fuming, $0.5 \mathrm{~mL}$ ( $1 \mathrm{~mL}$ was also tested) of concentrated $\mathrm{HCl}$ was pipetted into the bleach surrounding the inverted beaker. Immediately after $\mathrm{HCl}$ was added, the open dish of seeds was transferred into the jar to rest on top of the inverted beaker. The jar was sealed with the ball jar lid for $1 \mathrm{~h}$ (other durations were also tested). After $1 \mathrm{~h}$ (or other durations) elapsed, the dish was removed from the jar and immediately covered with the Petri dish lid.

Used solution was disposed of by diluting the bleach-HCl mixture into $\sim 2 \mathrm{~L}$ of tap water. The diluted solution was disposed down the sink with copious running water. Under a sterile laminar flow the dish with seeds was opened and allowed to off-gas for at least $30 \mathrm{~min}$. After off-gassing, the dish was sealed with Parafilm ${ }^{\circledR}$ until the seeds were used.

\section{Ethanol submersion}

The appropriate number of seeds were counted and $\sim 15 \%$ additional seeds were added. Seeds were placed in a $1.5 \mathrm{~mL}$ Eppendorf tube and $1 \mathrm{~mL}$ of $70 \%$ ethanol was added to the tube.
The tube was sealed and seeds were maintained submerged for $5 \mathrm{~min}$ (10 min was also tested). During the submersion, the seeds were vortex blended at least 10 times for $10 \mathrm{~s}$ each time. After the test duration, seeds were allowed to settle and excess ethanol was decanted under a sterile laminar flow. Using a sterile spatula, the seeds were scooped out of the Eppendorf tube and spread upon sterile filter paper in a sterile petri dish. With the petri dish open under laminar flow, excess ethanol was allowed to evaporate off for at least $30 \mathrm{~min}$. The dish containing seeds was sealed with Parafilm ${ }^{\circledR}$ until the seeds were used.

\section{Treatment confirmation}

Seed surface sterilization methods were confirmed by testing both seed germination and surface sterilization. For seed germination assessments, surface sterilized seeds and unsterilized controls were planted in Petri dishes on germination paper moistened with tap water until glistening and then sealed with two layers of Parafilm®. Sealed Petri dishes were placed in controlled environment chambers with low light $\left(\sim 50-150 \mu \mathrm{mol} \mathrm{m}{ }^{-2} \mathrm{~s}^{-1}\right.$ ) set to $20-23^{\circ} \mathrm{C}, 50-75 \%$ relative humidity (RH), and ambient $\mathrm{CO}_{2}$ at 500 $\mathrm{ppm}$. Seeds were tested immediately upon surface sterilization as well as approximately 1 month after sealed storage at room temperature. Surface sterilization was confirmed using two methods. The first involved direct plating of five treated seeds on Trypticase soy agar (TSA) medium with comparison of untreated controls for microbial growth. The second method used triplicate samples of 20 seeds (both treated and untreated) placed in $3 \mathrm{~mL}$ of sterile deionized $\mathrm{H}_{2} \mathrm{O}$ with glass beads. These were shaken for $2 \mathrm{~min}$ and plated onto TSA agar and Inhibitory mold agar (IMA). TSA plates were incubated at $30^{\circ} \mathrm{C}$ for $48 \mathrm{~h}$, and IMA cultures were maintained at $25^{\circ} \mathrm{C}$ for up to 7 days. Incubation was followed by enumeration of colony forming units (CFU). Methods for each seed type were only selected if they sterilized the surface of seeds and did not impede germination.

\section{Pillow Preparation}

Methods for pillow preparation were based on techniques used in the Biomass Production System (BPS) root module developed by ORBITEC (Morrow et al., 2001). Methods were established specifically for the volume and 
dimensions of the plant pillows designed to interface with the Veggie root mat reservoir. Both the Veggie plant pillows and root mat reservoirs were designed and constructed by ORBITEC (Madison, WI). Upon receipt of the pillows these were inspected in clean rooms at Kennedy Space Center. Inspection assessed the stitching and foam gaskets, and looked for sharp edges or insufficiently closed seams. Pillows were used if no sharp edges were observed, no openings where $600 \mu \mathrm{m}$ particles could escape were found, gasket cuts were level allowing substrates to be well contained, and pillow tops and bases were sewn in alignment. In the VEG-01 flight, two pillows were observed to not allow water to enter due to interior watering tubes clogged with tube material from the process of cutting drip holes on the tubes. Following this, air injection with a syringe was also used as an inspection criterion. The method of adding holes to the interior tubes was also modified to eliminate the potential for clogging. Any pillows that required it were reworked by ORBITEC. In preparation for flight, filled pillows were packaged in launch foam and subjected to simulated launch vibration testing at Kennedy Space Center (KSC) to confirm that these methods were sufficient to contain substrate and maintain seeds in planted positions. Vibration profiles based on launch vehicle measurements were programmed into vibrating tables and a Cargo Transfer Bag (CTB) packed with plant pillows was strapped to the table to mimic launch stowage conditions.

\section{Pillow filling}

All procedures were conducted under a sterile laminar flow using sterilized tools and plant pillows that had been constructed and maintained in a cleanroom environment. Pillow wicks were cut from polypropylene wipe material (KIMTECH PURE* W4 Dry Wipers, KimberlyClark Professional, Roswell, GA) as rectangles with dimensions of $23 \mathrm{~mm}$ x $120 \mathrm{~mm}$. The VEG01 and VEG-03 series flight experiments used standard CTBs designed to hold 18 pillows. Thus for each flight experiment 36 wicks were required, with an equal number needed for the ground control set of plant pillows. Pairs of wicks were placed together with one on top of the other with rougher or fuzzier sides of the wipe material facing inward. Wick pairs were then inserted through the slots of Veggie plant pillow foam gaskets (ORBITEC) with $18 \mathrm{~mm}$ of wick material left extending outside of the plant pillow (Figure 3A). Wick tails were adjusted with forceps inside the plant pillow, with the tails protruding through the interior watering ring and overlapping on the lower interior surface of the pillow (Figure 3B). Each internal tail of the pair of wicks was separated when filling the pillow with the arcillite fertilizer blend, to allow substrate to pass between the wicks inside of the pillow. Each pillow was filled with $250 \mathrm{~cm}^{3}$ of substrate by first adding $150 \mathrm{~cm}^{3}$ of substrate to a pillow, next adjusting the tails of the wicks to keep contact with the lower surface of the pillow and allow separation, and then adding the remaining $100 \mathrm{~cm}^{3}$ of substrate.

Following filling, the pillow open end was folded over a single time and temporarily sealed closed with lab tape leaving a tape tab. For the VEG-03 pillows, air flow through the pillow priming irrigation tube was again tested with a sterile $60 \mathrm{~mL}$ syringe and quick disconnect (QD) to make sure that the substrate had not clogged the interior drip line sufficient to create back pressure. Filled pillows were sewn shut with Nomex ${ }^{\circledR}$ thread by removing the tape, double folding the folded edge, and sewing a double seam along the center of the fold.

\section{Pillow planting}

After pillows were sewn they were stored in sterile reclosable bags (labeled with substrate type) until launch preparations commenced (i.e., shortly before turning them over for flight). The final steps in pillow preparation were to plant the surface sterilized seeds, add labels to the pillows, and seal up the planted pillows. For planting, 300 mg of Guar (a glue-like matrix, Sigma-Aldrich Corp., St. Louis, MO), was mixed with $25 \mathrm{~mL}$ of deionized $\mathrm{H}_{2} \mathrm{O}$. The mixture was stirred with a sterile spatula, microwaved on high for $10 \mathrm{~s}$, stirred again, microwaved on high for an additional $10 \mathrm{~s}$, and stirred once more. A $3 \mathrm{~mL}$ syringe with a 16 gauge needle was filled with the guar gum mixture. The syringe was allowed to come to room temperature and covered with foil to block light until it was used. Under sterile laminar flow, a sewn pillow was removed from its reclosable bag. With forceps, the paired wicks were opened and one seed was positioned. Seed 
positioning was performed to allow for radicle emergence into the interior of the pillow, so seeds were oriented with the tapered end pointing inward into the pillow interior (for lettuce or zinnia) or the seed scar (black spot) facing inward (for Chinese cabbage) so that the seed rested halfway into the pillow in the mid-plane of the foam gasket (Figure 3C). A drop of guar was used on the end of the forceps to help position the seed. A small drop of guar glue was used to attach the seed to the wick. The process was repeated with a second seed, positioning 8 to 10 $\mathrm{mm}$ from the first seed, with both seeds centered in the pair of wicks (Figure 3C). The wicks were pinched closed and held for approximately $20 \mathrm{~s}$. Each planted pillow was allowed to dry under sterile air flow for 20 min while the planting procedure was repeated with the remaining pillows. Forceps were sterilized between seed types. Flight pillow labels were inserted into Aclar ${ }^{\circledR}$ sleeves on the plant pillows after planting. Label material was selected specifically to be nontoxic and not off-gassing. After allowing guar to dry, each pillow was inserted, QD end first, into a gas impermeable Tedlar ${ }^{\circledR}$ bag (6.5 in $\mathrm{x} 8$ in Tedlar ${ }^{\circledR}$ bags, SKC Inc., Eighty Four, PA), while using care and folding flat the wicks to maintain seed position. The Tedlar ${ }^{\circledR}$ bag was heat sealed after most of the air was squeezed from the bag. The heat sealer used was a Polysealer model P200 (Fuji Impulse, Deerfield, IL) with a setting of 7.5 for the Tedlar ${ }^{\circledR}$ material. After sealing, a uniform seal without wrinkles was confirmed before the packaged pillow was removed from sterile air flow. Each sealed pillow was weighed and photographed. Figure 3D shows a packaged plant pillow prepared for flight.
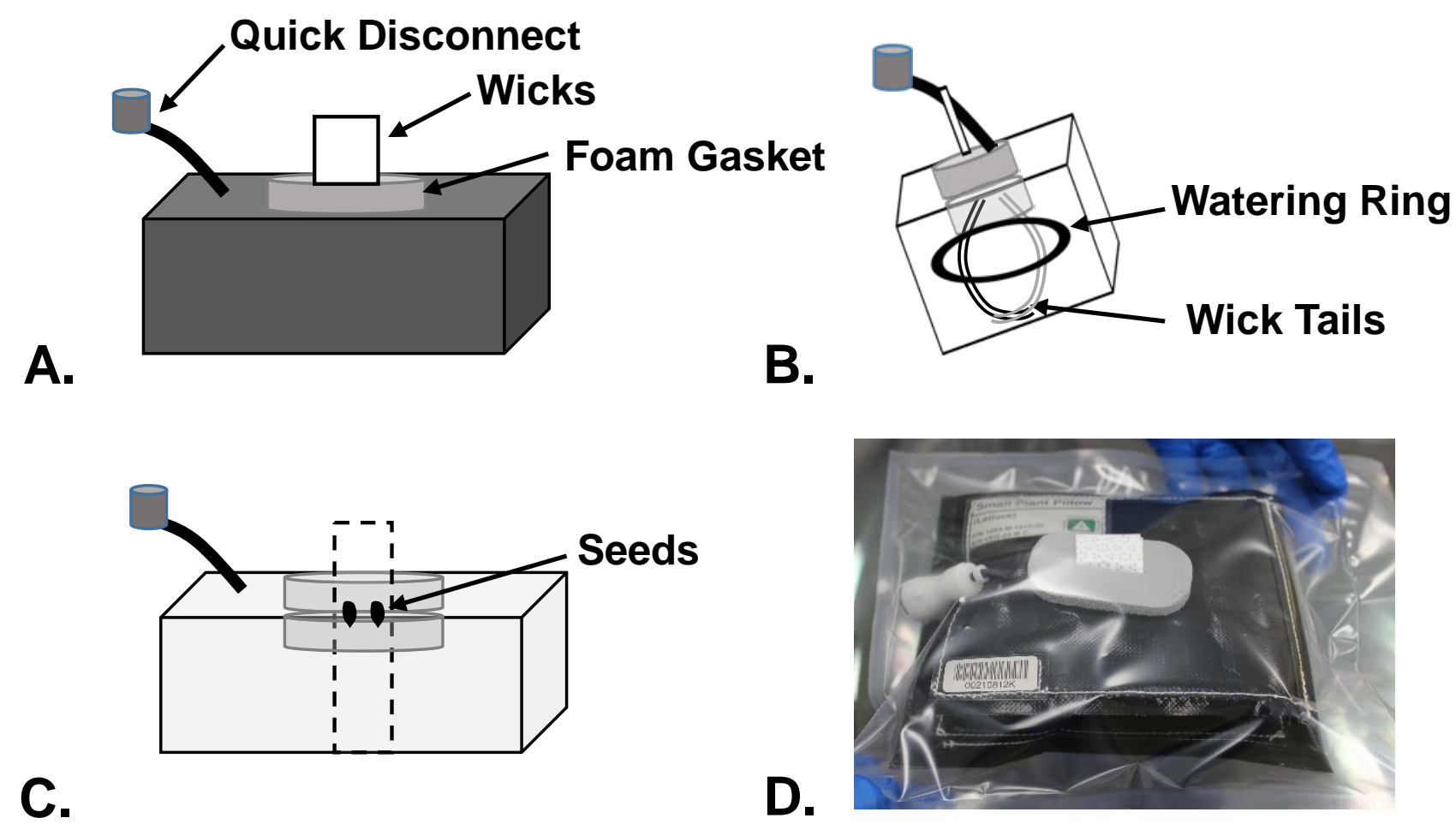

Figure 3. A. Schematic side view of a plant pillow. B. Schematic end cross sectional view of plant pillow. C. Schematic cut away side view of plant pillow showing seed placement. D. Photo of a planted pillow prepared for flight. 
Pillows were maintained under dry $(<50 \%$ $\mathrm{RH})$ and dark conditions prior to flight. Handling was minimized to reduce the capacity for substrate grinding and dust formation. Following sealing in Tedlar ${ }^{\circledR}$ bags, pillows were placed into custom-cut flight foam layered in a CTB and either transferred to a launch vehicle for flight or maintained in controlled laboratory conditions for ground controls.

\section{RESULTS}

\section{Substrate Preparation}

The first substrate cleaning method was time consuming, averaging around $1 \mathrm{~h}$ per $1000 \mathrm{~cm}^{3}$ batch using the deionized water system. While the number of substrate rinses required varied based on the particle size being washed and the dust level in a particular batch, cleaning of the $600 \mu \mathrm{m}$ to $1 \mathrm{~mm}$ particles averaged 9.7 rinses in the $30 \mathrm{~L}$ washing system. Cleaning of the 1-2 mm particles averaged 13.3 rinses with an overall average of 11.4 rinses for both particle sizes. This first method used a large quantity of deionized water (11.4 rinses uses an average of $274 \mathrm{~L}$ of water per $1000 \mathrm{~cm}^{3}$ of arcillite). Because of this, a second, less time consuming and more water efficient method was developed. For the second method, the average batch wash duration was 5 min per $500 \mathrm{~cm}^{3}$ of arcillite substrate and $19.4 \mathrm{~L}$ water was used. Per $1000 \mathrm{~cm}^{3}$ of arcillite the second substrate washing technique saved an average of $50 \mathrm{~min}$ and $235 \mathrm{~L}$ of water.

\section{Seed Preparation}

Preliminary testing with various seed sterilization methods demonstrated that the chlorine fuming method could be used to successfully surface sterilize lettuce seeds without inhibiting immediate and stored seed germination (Table 1). Initial tests were performed with a package of 'Waldmann's Green' lettuce seeds, which showed more contamination than the relatively clean 'Outredgeous' seeds used for flight. Bleach alone was insufficient to fume the seeds. When concentrated $\mathrm{HCl}$ was added to the bleach to produce chlorine gas, levels of $\mathrm{HCl}$ of 1 $\mathrm{mL}$ per $30 \mathrm{~mL}$ of bleach, or durations longer than one h, caused visible bleaching of lettuce seed coats and failure of seeds to germinate (Table 1). Treatment levels of $0.5 \mathrm{~mL}$ of $\mathrm{HCl}$ to $30 \mathrm{~mL}$ bleach allowed seed germination rates of 100\% and seed coats that were sterile. Seed germination rates also remained high after dry storage for 1 month with this method (Table 1).

The established chlorine fuming method was used successfully for both the 'Outredgeous' lettuce as well as for 'Tokyo Bekana' Chinese cabbage flight seeds (Table 2). However, for 'Profusion' zinnia and 'Durango' French marigold, chlorine fuming was not an effective treatment method (Table 2). An ethanol submersion technique was tested successfully for the zinnia seeds. Marigold seeds were not successfully treated with any of the methods tested, and thus, marigold was not selected for flight to ISS.

Table 1. Percentages of germination and microbial contamination in preliminary lettuce seed tests of fuming surface sterilization methods. Tests were conducted with 'Waldmann's Green' lettuce seeds that had microbial contamination. Germination was tested immediately and following 1 month of storage for promising treatments.

\begin{tabular}{lccc} 
Fuming Treatment & Germination & Contamination & Stored Germination \\
\hline Bleach alone-12 h & $96 \%$ & $100 \%$ & - \\
Bleach+1 mL HCL-20 h & $0 \%$ & $0 \%$ & - \\
Bleach+0.5 mL HCL-1h & $100 \%$ & $0 \%$ & $4 \%$ \\
Bleach+1 mL HCL-1h & $100 \%$ & $0 \%$ & $95 \%$ \\
Untreated & $96 \%-100 \%$ & $100 \%$ & $4 \%$
\end{tabular}


Table 2. Percentages of germination (G) and contamination (C) observed for Veggie candidate flight seeds tested with different surface sterilization treatments. Dashes indicate that these tests were not performed. Values in bold indicate methods selected for flight seeds.

\begin{tabular}{|c|c|c|c|c|c|c|c|c|}
\hline \multirow{2}{*}{ Treatment } & \multicolumn{2}{|c|}{$\begin{array}{l}\text { 'Profusion' } \\
\text { Zinnia }\end{array}$} & \multicolumn{2}{|c|}{$\begin{array}{c}\text { 'Durango' French } \\
\text { Marigold }\end{array}$} & \multicolumn{2}{|c|}{$\begin{array}{l}\text { 'Outredgeous' } \\
\text { Lettuce }\end{array}$} & \multicolumn{2}{|c|}{$\begin{array}{l}\text { 'Tokyo Bekana' } \\
\text { Chinese cabbage }\end{array}$} \\
\hline & G & C & G & C & G & C & G & C \\
\hline $\begin{array}{l}\text { Bleach + } 0.5 \mathrm{~mL} \\
\text { HCL - 1h }\end{array}$ & $80-100 \%$ & $0 \%$ & $70 \%$ & $40 \%$ & $100 \%$ & 0\% & $100 \%$ & 0\% \\
\hline $\begin{array}{l}\text { Bleach + } 1 \mathrm{~mL} \\
\text { HCL - 1h }\end{array}$ & $80 \%$ & $0 \%$ & $70 \%$ & $60 \%$ & - & - & $100 \%$ & $0 \%$ \\
\hline $\begin{array}{l}\text { Bleach + } 0.5 \mathrm{~mL} \\
\text { HCL - 5h }\end{array}$ & $30 \%$ & $0 \%$ & $10 \%$ & $0 \%$ & - & - & - & - \\
\hline $\begin{array}{l}70 \% \mathrm{EtOH}- \\
5 \mathrm{~min}\end{array}$ & $90-100 \%$ & 0\% & $30 \%$ & $60 \%$ & $80-100 \%$ & $20-40 \%$ & $100 \%$ & $100 \%$ \\
\hline $\begin{array}{l}70 \% \mathrm{EtOH}- \\
10 \mathrm{~min}\end{array}$ & - & - & - & - & - & - & $92 \%$ & $20 \%$ \\
\hline Untreated & $67-100 \%$ & $20-40 \%$ & $60-100 \%$ & $100 \%$ & $90-100 \%$ & $20-80 \%$ & $100 \%$ & $100 \%$ \\
\hline
\end{tabular}

\section{Pillow Preparation}

Plant pillows successfully passed preflight simulated launch testing on a vibration table. A small amount $\left(<0.5 \mathrm{~cm}^{3}\right)$ of arcillite substrate occasionally escaped the pillow gaskets upon vibration. Crew procedures were developed that call for the astronauts to have a vacuum cleaner available when opening Tedlar ${ }^{\circledR}$ bags to capture any loose arcillite. The VEG-01 plant pillows were planted and packaged in December, 2013, and these were launched to the ISS in April, 2014. The first set of lettuce was grown in May to June of 2014, the second set of lettuce was grown in July to August of 2015, while zinnias were grown in November 2015 to February 2016. VEG-03 plant pillows were prepared and launched to the ISS in February, 2016. Lettuce was grown October to December, 2016, while Chinese cabbage was grown January to February, 2017 and again April to May 2017. In all cases, seed germination was between $67 \%$ and $100 \%$ germination on the ISS and $75 \%$ to $100 \%$ in ground controls (Table 3). There were no obvious effects of storage conditions or duration on seed viability. Relatively poor germination in the
VEG-01B flight seeds was due to a pillow malfunction where water could not be added to one of the pillows due to clogged inner tubing. The VEG-01B ground test had one pillow with no seeds apparent; it was likely that one was missed in planting. VEG-01C also had one pillow with clogged tubing but water entered this pillow through the bottom surface allowing those seeds to germinate.

\section{DISCUSSION}

Veggie plant pillows showed good substrate containment and seed germination; however, the water delivery between the root mat reservoir and the plant pillows had challenges, with the root mat reservoir failing to provide sufficient water to meet the needs of the growing plants. Because of this, a contingency watering method of directly watering the plant pillows was implemented (Massa et al., 2017). While plant pillows with direct watering continue to be used and remain a viable method of growing plants in Veggie, the crew time demands of this method and the potential for over- or under-watering have led to research into different water delivery strategies. 
Table 3. Percentages of seeds germinating in the VEG-01 and VEG-03 tests. Each value represents a percentage of the 12 seeds planted in 6 pillows.

\begin{tabular}{|c|c|c|c|}
\hline Test & Plant & $\begin{array}{c}\text { Germination - } \\
\text { Flight (\%) }\end{array}$ & $\begin{array}{l}\text { Germination- } \\
\text { Ground (\%) }\end{array}$ \\
\hline VEG-01A & 'Outredgeous' lettuce & 75 & 100 \\
\hline VEG-01B & 'Outredgeous’ lettuce & $67 *$ & 75 \\
\hline VEG-01C & 'Profusion’ zinnia & $100 *$ & 92 \\
\hline VEG-03A & 'Outredgeous’ lettuce & 92 & 92 \\
\hline VEG-03B & 'Tokyo Bekana' Chinese cabbage & 92 & 100 \\
\hline VEG-03C & 'Tokyo Bekana' Chinese cabbage & 92 & 100 \\
\hline
\end{tabular}

For future growth studies, a modified water delivery system that will replace both the plant pillows and the root mat reservoir is being designed. While the plant growth hardware may change with the upgraded water delivery system, the lessons learned from substrate preparation, seed preparation, and pillow preparation will be translated into the next generation of Veggie hardware. Similar methods may also be used to prepare the science carrier for NASA's Advanced Plant Habitat, a fully automated plant growth chamber with over 180 sensors and control systems (Morrow et al., 2016). A second Veggie unit and the Advanced Plant Habitat will be installed on the ISS in the summer of 2017 and, along with the original Veggie, these will be available to the space biology research community to help advance knowledge of food crop production for space exploration.

\section{ACKNOWLEDGEMENTS}

This work was supported by NASA's Division of Space Life and Physical Sciences Research and Applications. Also we wish to thank the Veggie and VEG teams at KSC, ORBITEC, and at other NASA centers. Pillow preparation for VEG-01 and VEG-03 would not have been possible without the help of Matthew Romeyn, Matthew Mickens, LaShelle Spencer, Hannah Lyons, Jeff Richards, Stephanie Richards, Nicole Dufour, Deborah Dukes, Anne Marie Campbell, Gary Stutte, Bryan Onate, and Trent Smith. We also wish to thank Larry Koss who designed and built the second substrate washing system and Laura Reese who collected data for this manuscript. We are grateful to Matt Romeyn for advice on the manuscript. The authors wish to thank Profile Products LLC for providing sieved substrate and Florikan for providing advice on fertilizer usage.

\section{REFERENCES}

Clough S, Bent A (2011) Vapor-Phase Sterilization of Arabidopsis Seed. From http://www.plantpath.wisc.edu/fac/afb/vapst er.html; accessed on 09/02/2016

Graham T, Scorza R, Wheeler R, Smith B, Dardick C, Dixit A, Raines D, Callahan A, Srinivasan C, Spencer L, Richards J, Stutte G (2015) Over-expression of FT1 in plum (Prunus domestica) results in phenotypes compatible with spaceflight: A potential new candidate crop for bioregenerative life 
support systems. Gravitational and Space Research 3: 39-50

Jones SB, Or D, Bingham GE, Morrow RC (2002) ORZS: Optimization of Root Zone Substrates for Microgravity. 32nd International Conference on Environmental Systems, SAE Technical Paper 2002-012380

Maloof JN (2014) Ethanol Seed Sterilization. From

http://openwetware.org/wiki/Maloof_Lab:S eed_Sterilization; accessed on 09/02/2016

Massa, GD, Dufour NF, Carver JA, Hummerick ME, Wheeler RM, Morrow RC, Smith TM (2017) VEG-01: Veggie hardware validation testing on the International Space Station. Open Agriculture 2: 33-41

Massa GD, Newsham G, Hummerick ME, Caro JL, Stutte GW, Morrow RC, Wheeler RM (2013) Preliminary species and media selection for the Veggie space hardware. Gravitational and Space Research 1: 95106

Massa GD, Wheeler RM, Morrow RC, Levine HG (2016) Growth chambers on the International Space Station for large plants. Acta Horticulturae 1134: 215-222

Morrow R, Richter R, Tellez G, Monje O, Wheeler R, Massa G, Dufour N, Onate B (2016) A New Plant Habitat Facility for the ISS. 46th International Conference on Environmental Systems, ICES paper 2016320

Morrow RC, Bula RJ, Tibbitts TW, Dinauer WR (1994) The Astroculture ${ }^{\mathrm{TM}}$ flight experiment series, validating technologies for growing plants in space. Advances in Space Research 14: 29-37

Morrow RC, Crabb TM (2000) Biomass Production System (BPS) plant growth unit. Advances in Space Research 26: 289-298

Morrow RC, Crabb TM, Iverson JT, Frank JG (2001) Science Accomodations in the Biomass Production System. 31st International Conference on Environmental Systems, SAE Technical Paper 2001-012231

Morrow RC, Remiker RW (2009) A Deployable Salad Crop Production System for Lunar Habitats. 39th International Conference on Environmental Systems, SAE Technical Paper 2009-01-2382

Morrow RC, Remiker RW, Mischnick MJ, Tuominen LK, Lee MC, Crabb TM (2005) A Low Equivalent System Mass Plant Growth Unit for Space Exploration. 35th International Conference on Environmental Systems, SAE Technical Paper 2005-012843

Norikane JH, Jones SB, Steinberg SL, Levine HG, Or D (2005) Porous media matric potential and water content measurements during parabolic flight. Habitation 10: 117-126

Stutte GW, Newsham G, Morrow RC, Wheeler RM (2011) Concept for Sustained Plant Production on ISS Using VEGGIE Capillary Mat Rooting System. 41st International Conference on Environmental Systems, International Conference on Environmental Systems, AIAA Technical Paper No. 2011-5263 